\title{
A new compression algorithm for small data communication in wireless sensor network
}

\begin{abstract}
Energy consumption is one of the most critical issues in wireless sensor network (WSN). For a sensor device, transmission of data is considered as the most energy consuming task, and it mostly depends on the size of the data. Fortunately, data compression can be used to minimise the transmitted data size and thus extend sensor's lifetime. In this paper, we propose a new lossless compression algorithm that can handle small data communication in WSNs. Using compression ratio, memory usage, number of instructions and execution speed as a comparison parameters, the proposed algorithm is measured against a set of existing algorithms. Two different datasets have been used for this purpose; namely, self-generated dataset and real sensor dataset from Harvard Sensor Library. As a result, the proposed algorithm not only outclasses other existing algorithms but most importantly produces positive compression ratio throughout the whole test where most existing algorithms experience an expansion in data size when dealing with very small data.
\end{abstract}

Keyword: Lossless compression; Dictionary-based algorithm; Data correlation; m-LZO; Gzip; Huffman coding; LZ77; LZMA; S-LZW 